\title{
A Distinct Pattern of Circulating Amino Acids Characterizes Older Persons with Physical Frailty and Sarcopenia: Results from the BIOSPHERE Study
}

\author{
Riccardo Calvani ${ }^{1,2}\left(\mathbb{0}\right.$, Anna Picca ${ }^{1,2, *(0)}$, Federico Marini ${ }^{3}(0)$, Alessandra Biancolillo ${ }^{3}$, \\ Jacopo Gervasoni 1,2, Silvia Persichilli 1,2, Aniello Primiano ${ }^{2}$, Hélio José Coelho-Junior ${ }^{2,4}$ (D), \\ Maurizio Bossola 1,2, Andrea Urbani 1,2 , Francesco Landi 1,2, Roberto Bernabei ${ }^{1,2}$ and \\ Emanuele Marzetti ${ }^{1}$ \\ 1 Fondazione Policlinico Universitario Agostino Gemelli, IRCCS, Rome 00168, Italy; \\ riccardo.calvani@gmail.com (R.C.); jacopo.gervasoni@policlinicogemelli.it (J.G.); \\ silvia.persichilli@policlinicogemelli.it (S.P.); mauriziobossola@gmail.com (M.B.); \\ andrea.urbani@unicatt.it (A.U.); francesco.landi@unicatt.it (F.L.); \\ roberto.bernabei@unicatt.it (R.B.); emanuele.marzetti@policlinicogemelli.it (E.M.) \\ 2 Università Cattolica del Sacro Cuore, Rome 00168, Italy; anielloprim@gmail.com (A.P.); \\ coelhojunior@hotmail.com.br (H.J.C.-J.) \\ 3 Department of Chemistry, Sapienza University of Rome, Rome 00168, Italy; \\ federico.marini@uniroma1.it (F.M.); alessandra.biancolillo@uniroma1.it (A.B.) \\ 4 Applied Kinesiology Laboratory-LCA, School of Physical Education, University of Campinas, \\ Campinas-SP 13.083-851, Brazil \\ * Correspondence: anna.picca1@gmail.com; Tel.: +39-06-3015-5559
}

Received: 17 September 2018; Accepted: 1 November 2018; Published: 6 November 2018

\begin{abstract}
Physical frailty and sarcopenia (PF\&S) are hallmarks of aging that share a common pathogenic background. Perturbations in protein/amino acid metabolism may play a role in the development of PF\&S. In this initial report, 68 community-dwellers aged 70 years and older, 38 with $\mathrm{PF} \& S$ and 30 non-sarcopenic, non-frail controls (nonPF\&S), were enrolled as part as the "BIOmarkers associated with Sarcopenia and Physical frailty in EldeRly pErsons" (BIOSPHERE) study. A panel of 37 serum amino acids and derivatives was assayed by UPLC-MS. Partial Least Squares-Discriminant Analysis (PLS-DA) was used to characterize the amino acid profile of PF\&S. The optimal complexity of the PLS-DA model was found to be three latent variables. The proportion of correct classification was $76.6 \pm 3.9 \%$ ( $75.1 \pm 4.6 \%$ for enrollees with PF\&S; $78.5 \pm 6.0 \%$ for nonPF\&S). Older adults with PF\&S were characterized by higher levels of asparagine, aspartic acid, citrulline, ethanolamine, glutamic acid, sarcosine, and taurine. The profile of nonPF\&S participants was defined by higher concentrations of $\alpha$-aminobutyric acid and methionine. Distinct profiles of circulating amino acids and derivatives characterize older people with PF\&S. The dissection of these patterns may provide novel insights into the role played by protein/amino acid perturbations in the disabling cascade and possible new targets for interventions.
\end{abstract}

Keywords: aging; muscle; protein; metabolism; metabolomics; profiling; biomarkers; multi-marker; physical performance; multivariate

\section{Introduction}

Over the last decades, sarcopenia, the progressive and generalized decline in skeletal muscle mass and function with age, has become a "blockbuster" condition in geriatrics, given its increasing prevalence in a globally aging world and its clinical relevance [1-4]. Indeed, this condition conveys 
a broad spectrum of negative health-related outcomes, including disability, loss of independence, institutionalization, and mortality [5,6]. Frailty has been defined as a geriatric "multidimensional syndrome characterized by decreased reserve and diminished resistance to stressors," and is often envisioned as a pre-disability condition [7]. Sarcopenia overlaps with the clinical picture of frailty, especially in its physical domain, and may represent both the biological substratum of physical frailty (PF) and the pathophysiologic basis upon which the negative health outcomes of PF develop [8,9]. The two conditions have therefore been merged into a new entity (i.e., PF and sarcopenia; PF\&S) [10] that was operationalized in the context of the "Sarcopenia and Physical fRailty IN older people: multi-componenT Treatment strategies" (SPRINTT) project [11,12].

Although the pathophysiology of PF\&S is complex and multifactorial, the central role attributed to muscle wasting suggests that biomarkers related to sarcopenia may be used to support the diagnosis and track the evolution of PF\&S, unveil its underlying mechanisms, and identify meaningful targets for interventions $[13,14]$.

Dietary protein intake and circulating amino acids play a pivotal role in muscle plasticity and trophism [15], but also modulate several biological processes (including inflammation, insulin sensitivity, and redox homeostasis) that may be involved in age-related muscle atrophy and dysfunction $[16,17]$. Hence, perturbations in protein-amino acid metabolism may represent a major mechanism in sarcopenia $[18,19]$.

Amino acid profiling, especially when coupled with multivariate statistical analysis, may serve as a powerful analytical approach to explore the possible role of protein-amino acid networks in PF\&S [20]. Recently, distinct amino acid signatures were associated with muscle mass in older adults with functional limitations [21] and low muscle quality [22] in the Baltimore Longitudinal Study of Aging. Moreover, reduced non-fasting plasma concentrations of the branched-chain amino acids (BCAAs) leucine and isoleucine were detected in Norwegian older community-dwellers with sarcopenia [23], while higher proline concentrations were independently associated with sarcopenia in older Japanese people [24]. Finally, low plasma levels of essential amino acids (EAAs) characterized the amino acid profile of severely frail Japanese older people compared with non-frail peers [25].

The "BIOmarkers associated with Sarcopenia and Physical frailty in EldeRly pErsons" (BIOSPHERE) study was designed to determine and validate a panel of PF\&S biomarkers encompassing systemic inflammation, oxidative stress, muscle remodeling, neuromuscular junction dysfunction, and amino acid metabolism through multivariate statistical modeling [26]. In the present work, we report the initial results obtained through the simultaneous analysis of an array of circulating amino acids and derivatives coupled with Partial Least Squares-Discriminant Analysis (PLS-DA). This innovative approach allowed identifying distinct patterns of circulating amino acids and derivatives that characterize older adults with and without PF\&S. This may represent a first relevant step towards the integration of specific biochemical measurements into the assessment of $\mathrm{PF} \& S$ in research and clinical settings.

\section{Materials and Methods}

\subsection{Study Design and Population}

BIOSPHERE was conceived as a cross-sectional, case-control study [26]. The study protocol was approved by the Ethics Committee of the Università Cattolica del Sacro Cuore (Rome, Italy; protocol number: 8498/15) and is thoroughly described elsewhere [26]. Briefly, after obtaining written informed consent, 200 older persons, 100 cases (individuals with PF\&S) and 100 non-physically frail, non-sarcopenic (nonPF\&S) controls aged 70+ were enrolled. Selection criteria are reported in Table S1. Candidates were diagnosed with PF\&S when presenting the following parameters: (a) low physical performance, defined as a summary score on the Short Physical Performance Battery (SPPB) [27] between 3 and 9; (b) low appendicular muscle mass (aLM) according to the criteria recommended by the Foundation for the National Institutes of Health (FNIH) sarcopenia project [28]; and (c) absence of 
major mobility disability, operationalized as an inability to walk $400 \mathrm{~m}$ in $15 \mathrm{~min}$ at a usual pace [29]. This initial analysis involved 68 participants (38 cases and 30 controls) in whom circulating amino acids and derivatives were measured.

\subsection{Measurement of Appendicular Lean Mass by Dual X-Ray Absorptiometry (DXA)}

Whole-body DXA scans were obtained on a Hologic Discovery A densitometer (Hologic, Inc., Bedford, MA, USA). Scan acquisition and analysis were performed according to manufacturer's directions. Candidates were considered to be eligible if presenting with an aLM to body mass index (BMI) ratio $\left(\mathrm{aLM}_{\mathrm{BMI}}\right)<0.789$ or $<0.512$ in men and women, respectively. When the aLM $\mathrm{BMI}_{\mathrm{C}}$ criterion was not met, candidates were tested with the alternative criterion (i.e., crude aLM $<19.75 \mathrm{~kg}$ in men and $<15.02 \mathrm{~kg}$ in women) [28].

\subsection{Blood Sample Collection}

Blood samples were collected in the morning by venipuncture of the median cubital vein after overnight fasting, using commercial collection tubes (BD Vacutainer ${ }^{\circledR}$; Becton, Dickinson and Co., Franklin Lakes, NJ, USA). For serum separation, samples were left at room temperature for $20 \mathrm{~min}$ and subsequently centrifuged at $1000 \times g$ for $10 \mathrm{~min}$ at $4^{\circ} \mathrm{C}$. Aliquots of serum were subsequently stored at $-80{ }^{\circ} \mathrm{C}$ until analysis.

\subsection{Amino Acids Profiling}

Thirty-seven amino acids and derivatives (1-methylhistidine, 3-methylhistidine, 4-hydroxyproline, $\alpha$-aminobutyric acid, $\beta$-alanine, $\beta$-aminobutyric acid, $\gamma$-aminobutyric acid, alanine, aminoadipic acid, anserine, arginine, asparagine, aspartic acid, carnosine, citrulline, cystathionine, cystine, ethanolamine, glutamic acid, glycine, histidine, isoleucine, leucine, lysine, methionine, ornithine, phenylalanine, phosphoethanolamine, phosphoserine, proline, sarcosine, serine, taurine, threonine, tryptophan, tyrosine, valine) were measured in serum through a ultraperformance liquid chromatography/mass spectrometry (UPLC/MS) validated methodology. Briefly, $50 \mu \mathrm{L}$ of sample were mixed with $100 \mu \mathrm{L}$ $10 \%(w / v)$ sulfosalicylic acid containing an internal standard mix $(50 \mu \mathrm{M})$ (Cambridge Isotope Laboratories, Inc., Tewksbury, MA, USA) and centrifuged at $1000 \times g$ for $15 \mathrm{~min}$. Ten microliters of the supernatant were transferred into a vial containing $70 \mu \mathrm{L}$ of borate buffer to which $20 \mu \mathrm{L}$ of AccQ Tag reagents (Waters Corporation, Milford, MA, USA) were subsequently added. Samples were then vortexed for $10 \mathrm{~s}$ and heated at $55^{\circ} \mathrm{C}$ for $10 \mathrm{~min}$. The chromatographic separation was performed by ACQUITY H-Class (Waters Corporation) using a CORTECS UPLC C18 column $1.6 \mu \mathrm{m} 2.1 \times 150 \mathrm{~mm}$ (Waters Corporation) eluted at a flow rate of $500 \mu \mathrm{L} / \mathrm{min}$ with a linear gradient (9 min) from 99 to 1 water $0.1 \%$ formic acid in acetonitrile $0.1 \%$ formic acid. The mass spectrometer was an ACQUITY QDa single quadrupole equipped with electrospray source operating in positive mode (Waters Corporation). The analytical process was monitored using amino acid controls (level 1 and level 2) manufactured by the MCA laboratory of the Queen Beatrix Hospital (Winterswijk, The Netherlands).

\subsection{Statistical Analysis}

All analyses were performed using in-house routines running under MATLAB R2015b environment (The MathWorks, Natick, MA, USA).

\subsubsection{Descriptive Statistics}

Differences in demographic, anthropometric, clinical, and functional characteristics between cases and controls were assessed via $t$-test statistics and $\chi^{2}$ or Fisher exact tests, for continuous and categorical variables, respectively. All tests were two-sided, with statistical significance set at $p<0.05$. 


\subsubsection{Partial Least Squares-Discriminant Analysis}

The strategy for the identification and validation of potential biomarkers for PF\&S relied on the building of discriminant models to differentiate cases from controls. The approach chosen for the present study was based on PLS-DA [30], because of its versatility and ability to deal with highly correlated predictors. Briefly, PLS-DA is a classification method based on the PLS regression algorithm [31]. PLS-DA builds the linear relation between a set of responses $Y$ and a matrix of predictors $X$ by projecting the latter onto a low-dimensional space of latent (abstract) variables (LVs) that are characterized by having the highest covariance with the responses to be predicted. The statistical reliability of the PLS-DA model was subsequently verified by a double cross-validation (DCV) procedure and by means of randomization tests [32]. Three figures of merit were considered in the present study: (i) the number of misclassifications (NMC); (ii) the area under the receiver operating characteristic (ROC) curve (AUROC); and (iii) the value of the discriminant Q2 (DQ2) [33].

For the identification of potential biomarkers, two approaches aimed at highlighting the experimental variables contributing the most to the classification model were followed, and they involved inspecting variable importance in projection (VIP) indices [31] and rank product (RP) [34], respectively. A more detailed description of the PLS-DA statistics is provided as supplementary material.

\section{Results}

\subsection{Descriptive Characteristics of the Study Population}

The study population included 38 older adults with PF\&S and 30 nonPF\&S controls. The main demographic, anthropometric, clinical, and functional characteristics of the study population according to the presence of PF\&S are presented in Table 1. No differences between groups were observed with regard to age, gender distribution, number of co-morbid conditions, and number of prescription medications. The distribution of specific disease conditions and the prevalence of use of individual drug classes are shown in Table S2. As expected, physical performance, as assessed by the SPPB, was lower in PF\&S participants (SPPB score: $7.4 \pm 1.5)$ relative to controls $(11.3 \pm 0.9)(p<0.0001)$. Similarly, aLM, either absolute or adjusted for BMI, was smaller in the PF\&S group compared with nonPF\&S enrollees.

Table 1. Main characteristics of BIOmarkers associated with Sarcopenia and Physical frailty in EldeRly pErsons (BIOSPHERE) participants according to the presence of physical frailty and sarcopenia (PF\&S).

\begin{tabular}{lccc}
\hline & PF\&S $(\boldsymbol{n}=\mathbf{3 8})$ & nonPF\&S $(\boldsymbol{n}=\mathbf{3 0})$ & $\boldsymbol{p}$ \\
\hline Age, years (mean \pm SD) & $76.4 \pm 4.9$ & $74.6 \pm 4.3$ & 0.1067 \\
Gender (female), $n(\%)$ & $25(65.8)$ & $16(53.3)$ & 0.4280 \\
BMI, kg/m ${ }^{2}$ (mean \pm SD) & $29.1 \pm 4.4$ & $26.7 \pm 2.4$ & 0.0112 \\
SPPB (mean \pm SD) & $7.4 \pm 1.5$ & $11.3 \pm 0.9$ & $<0.0001$ \\
aLM, kg (mean \pm SD) & $16.2 \pm 3.2$ & $19.4 \pm 3.9$ & 0.0004 \\
aLM & $0.554 \pm 0.120$ & $0.795 \pm 0.264$ & $<0.0001$ \\
Number of disease conditions * (mean \pm SD) & $2.3 \pm 1.5$ & $1.8 \pm 1.4$ & 0.1448 \\
Number of medications (mean \pm SD) & $3.2 \pm 1.8$ & $2.8 \pm 1.9$ & 0.4115 \\
\hline
\end{tabular}

${ }^{*}$ Includes hypertension, coronary artery disease, prior stroke, peripheral vascular disease, diabetes, chronic obstructive pulmonary disease, and osteoarthritis. BMI: body mass index; SPPB: Short Physical Performance Battery; aLM: appendicular lean mass; PF\&S: physical frailty and sarcopenia; nonPF\&S: non physically frail, non sarcopenic; SD: standard deviation.

\subsection{Participant Classification According to PLS-DA}

In order to verify the existence of a specific pattern of amino acids in participants with PF\&S, a PLS-DA classification model was constructed and validated. The optimal PLS-DA model was built using three LVs that accounted for more than $44 \%$ of the variance originally present in the $\mathrm{X}$ block. As indicated by the DCV procedure, the model allowed to correctly predict the presence of PF\&S in 
$95.7 \pm 2.1 \%$ of participants in the calibration phase ( $94.7 \pm 3.8 \%$ for PF\&S and $96.7 \pm 4.6 \%$ for controls), $84.1 \pm 2.7 \%$ in the internal validation stage $(82.6 \pm 3.6 \%$ for PF\&S and $86.0 \pm 4.8 \%$ for controls), and $76.6 \pm 3.9 \%$ in external validation $(75.1 \pm 4.6 \%$ for PF\&S; $78.5 \pm 6.0 \%$ for nonPF\&S). Figure 1 , which depicts the projection of participants onto the space spanned by the first two LVs of the PLS-DA model, shows a clear separation between participants with and without PF\&S.

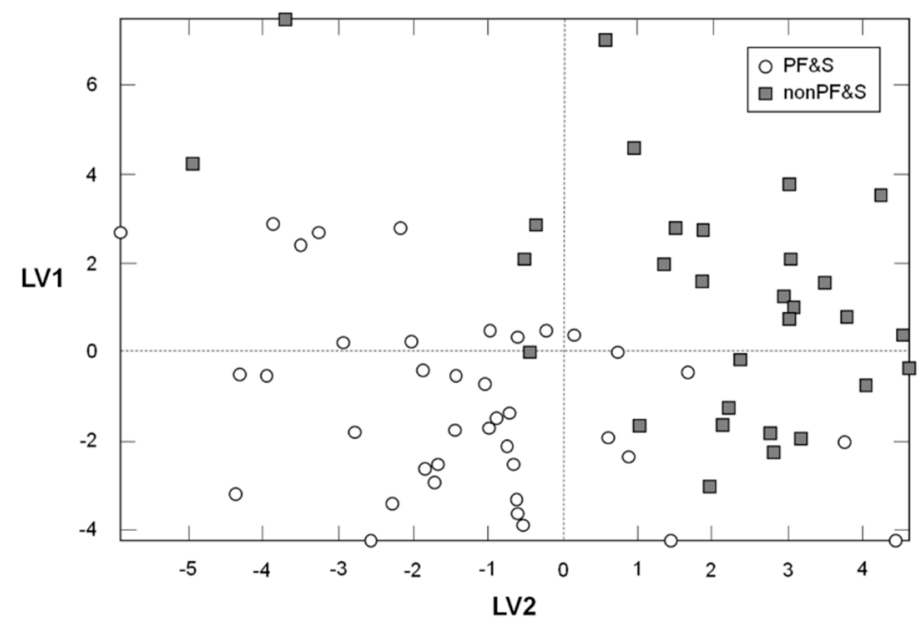

Figure 1. Scores plot showing the separation of participants according to the serum concentrations of amino acids and derivatives in the space spanned by the two latent variables (LV1 and LV2), as determined by Partial Least Squares-Discriminant Analysis (PLS-DA).

The classification ability of the PLS-DA model was further validated by comparing the results of the DCV with the distributions of NMC, AUROC and DQ2 under the null hypothesis (Figure 2). For each of the three figures of merits considered, the values obtained on the real dataset fell outside of the corresponding null hypothesis distribution, which corresponds to a $p<0.05$.
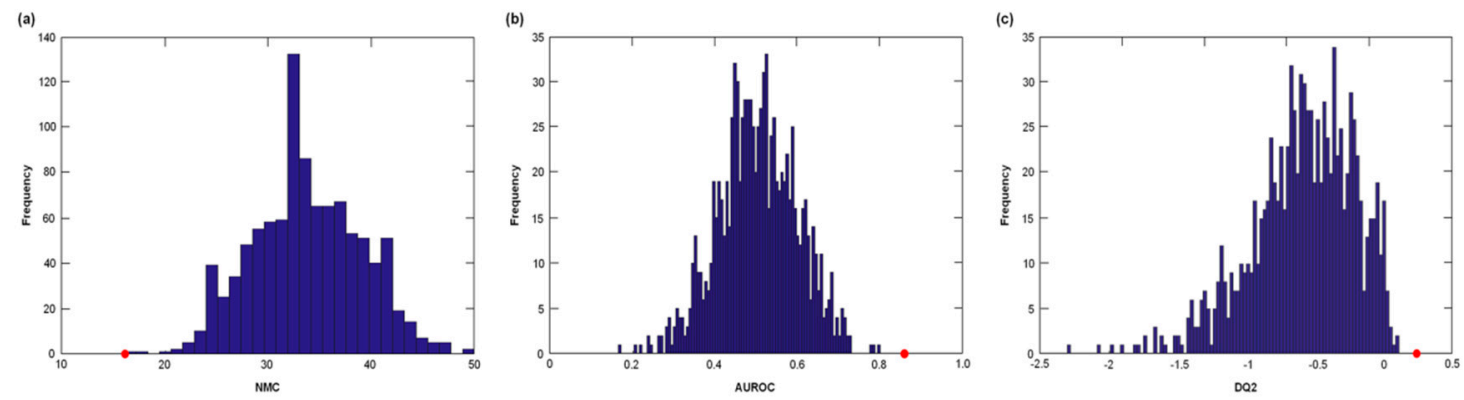

Figure 2. Distribution of (a) number of misclassifications (NMC), (b) area under the receiver operating characteristic (ROC) curve (AUROC), and (c) discriminant Q2 (DQ2) values under their respective null hypothesis as estimated by permutation tests (blue histograms) and the corresponding values obtained by the PLS-DA model on unpermuted data (red circles). Values obtained on the real dataset (red circles) fall outside of the corresponding null hypothesis distribution (blue histograms), corresponding to a $p<0.05$.

In order to identify the metabolites that were mostly involved in discriminating between cases and controls, the values of the VIP indices were inspected. The variables corresponding to a VIP greater than one are reported in Table 2. Nine amino acids were found to contribute significantly to the discrimination model. Participants with PF\&S were characterized by higher levels of asparagine, aspartic acid, citrulline, ethanolamine, glutamic acid, sarcosine, and taurine. Conversely, the profile of non-PF\&S individuals was defined by higher levels of $\alpha$-aminobutyric acid (AABA) and methionine. Serum concentrations of non-discriminant amino acids are reported in Table S3. 
Table 2. Serum concentrations of discriminant analytes, variable importance in projection (VIP) values, and rank product (RP) values in BIOSPHERE participants with and without physical frailty and sarcopenia (PF\&S). Serum concentrations are shown as mean \pm standard deviation.

\begin{tabular}{lcccc}
\hline & PF\&S $(\boldsymbol{n}=\mathbf{3 8})$ & nonPF\&S $(\boldsymbol{n}=\mathbf{3 0})$ & VIP & RP \\
\hline$\alpha$-aminobutyric acid $(\mu \mathrm{mol} / \mathrm{L})$ & $20.0 \pm 4.9$ & $22.3 \pm 5.7$ & 2.2 & 8.0 \\
Asparagine $(\mu \mathrm{mol} / \mathrm{L})$ & $91.0 \pm 12.6$ & $77.8 \pm 13.4$ & 3.4 & 2.0 \\
Aspartic Acid $(\mu \mathrm{mol} / \mathrm{L})$ & $24.6 \pm 5.4$ & $17.0 \pm 4.0$ & 5.8 & 2.6 \\
Citrulline $(\mu \mathrm{mol} / \mathrm{L})$ & $44.8 \pm 12.1$ & $36.8 \pm 11.5$ & 2.1 & 2.8 \\
Ethanolamine $(\mu \mathrm{mol} / \mathrm{L})$ & $10.3 \pm 1.7$ & $9.0 \pm 2.2$ & 1.7 & 9.9 \\
Glutamic acid $(\mu \mathrm{mol} / \mathrm{L})$ & $71.7 \pm 16.6$ & $54.3 \pm 21.2$ & 2.3 & 8.5 \\
Methionine $(\mu \mathrm{mol} / \mathrm{L})$ & $22.6 \pm 2.8$ & $23.4 \pm 5.7$ & 1.3 & 6.3 \\
Sarcosine $(\mu \mathrm{mol} / \mathrm{L})$ & $1.9 \pm 0.6$ & $1.5 \pm 0.5$ & 1.4 & 8.0 \\
Taurine $(\mu \mathrm{mol} / \mathrm{L})$ & $220.1 \pm 36.5$ & $189.5 \pm 47.2$ & 1.8 & 6.7 \\
\hline
\end{tabular}

\section{Discussion}

In the present study, we report the first results from the BIOSPHERE study. The most relevant finding was that older individuals with PF\&S showed a distinct profile of circulating amino acids characterized by higher serum levels of asparagine, aspartic acid, citrulline, ethanolamine, glutamic acid, sarcosine, and taurine. Conversely, the profile of nonPF\&S participants was defined by higher levels of AABA and methionine.

The existence of an amino acid signature in the setting of PF\&S suggests that specific metabolic alterations might be involved in the pathogenesis of this condition. Indeed, PF\&S was associated with lower circulating levels of the EAA methionine. EAAs are defined as those amino acids that must be provided with the diet to meet optimal requirements [35]. The reduction of serum concentrations of a number of EAAs (including methionine) with age was reported in both genders and was purportedly associated with decreases in total energy and protein intake [36]. In addition, low plasma levels of EAA were found in severely frail older people [25]. These findings may be linked to malnutrition (both quantitative and qualitative), a common causative factor of frailty and sarcopenia [37,38]. The concomitant low serum concentration of the non-essential non-proteinogenic amino acid AABA seems to corroborate the previous finding since AABA may derive from the catabolism of methionine [39]. Furthermore, plasma levels of AABA were found to be associated with both the quality and amount of dietary protein [40,41]. Although these findings seem to point towards a poor-quality protein diet or (selective) malabsorption, further studies are needed to clarify the relationship between diet and circulating EAA levels in the context of PF\&S.

Methionine is also involved in one-carbon metabolism, a crucial pathway that modulates multiple physiologic processes, including nucleotide biosynthesis, amino acid homeostasis, epigenetic maintenance, and redox balance [42]. Not surprisingly, alterations in one-carbon metabolism were observed in aging and age-related diseases, such as cancer, cardiovascular disease, and neurodegeneration [42,43]. Sarcosine, the $N$-methyl-derivative of glycine, is another relevant intermediate of one-carbon metabolism [42]. Sarcosine is formed from dietary choline and the metabolism of methionine [44,45], and can be found in muscles and other body tissues. A recent metabolomics study showed that circulating sarcosine levels were reduced with aging both in rodents and humans, while dietary restriction prevented this decline in both species [46]. Counterintuitively, sarcosine levels were higher in persons with PF\&S relative to controls. However, circulating sarcosine may increase in case of folate deficiency, because folate mediates the conversion of sarcosine to glycine [45]. Thus, this finding might be linked to insufficient folate ingestion and/or perturbation in folate/one-carbon metabolism.

Sarcosine also activates autophagy in mouse fibroblasts in a dose-dependent manner [46], and alterations in myocyte quality control mechanisms (including autophagy) may contribute to sarcopenia [47-49]. In particular, defective autophagic clearance of damaged cellular constituents, alterations in mitochondrial proteostasis and dynamics, and impaired mitochondriogenesis are thought 
to be critically involved in age-related muscle degeneration [50]. In this context, the presence of ethanolamine among the most discriminant metabolites for PF\&S classification is of particular interest. Ethanolamine is a naturally occurring amino alcohol that plays a pivotal role in the synthesis of phosphatidylethanolamine, a central intermediate of lipid metabolism and a major component of biological membranes [51]. Phosphatidylethanolamine is also directly involved in the regulation of autophagy [52], and it is postulated that ethanolamine treatment or the consumption of ethanolamine-rich foods may increase cellular phosphatidylethanolamine levels, induce autophagy, and provide beneficial anti-aging effects across species [52]. While serum ethanolamine levels were different between PF\&S and controls, this did not result in a corresponding difference in serum phosphatidylethanolamine concentrations, suggesting alterations in CDP-ethanolamine pathway, the major route of phosphatidylethanolamine production [53]. Interestingly, the disruption of CDP-ethanolamine pathway in muscle was associated with alterations in mitochondrial biogenesis and muscle atrophy in mice [54].

Taurine is a ubiquitous non-proteinogenic sulfur-containing amino acid that represents the most abundant free amino acid in the heart, retina, skeletal muscle, brain, and leukocytes, accounting for approximately $0.1 \%$ of total body weight [55]. In skeletal muscle, which contains $70 \%$ of total body taurine, this amino acid is involved in the regulation of ion channel function, membrane stability, mitochondrial quality control, and calcium homeostasis [56-59]. In muscle, taurine also serves osmoregulatory, anti-oxidant, and anti-inflammatory functions [56-59]. Given these multiple actions, taurine has recently been proposed as a candidate therapeutic agent against sarcopenia [60]. While it is reported that serum taurine concentrations decline with age in men [36], increased levels of serum taurine have been retrieved in the metabolic profiles of old wild-type mice from different genetic backgrounds [61]. Circulating levels of taurine are regulated by the balance among different factors, including dietary intake, intestinal absorption, bile acid conjugation, urinary excretion, and endogenous synthesis from methionine and cysteine [55]. Taurine may be released from cells following osmotic perturbations, oxidative stress, and (chronic) inflammatory stimulation [58]. Further studies are needed to unveil the mechanisms responsible for the high circulating taurine levels observed in older adults with PF\&S.

Citrulline is a non-essential non-protein amino acid with a key role in nitrogen homeostasis [62]. Citrulline is an end product of glutamine metabolism and an endogenous precursor of arginine [63]. For its capacity of promoting endothelial nitric oxide availability and vasodilation, "sparing" arginine and glutamine from hepatic catabolism and the supposed ability to activate mTORC1 signaling [64], citrulline was proposed as a pharmaconutrient to counteract sarcopenia [65]. Several reports have shown that serum citrulline increases with age $[36,66,67]$. In addition, in a metabolomics study assessing the individual variability in human blood metabolites [68], citrulline was among the circulating molecules that exhibit a remarkable age-related increase. The authors attributed this finding to impairment in urea cycle efficiency due to the progressive decline of liver and renal function with age [68]. However, no differences in kidney or liver function were observed between participants belonging to the two BIOSPHERE study groups. Further investigation on interorgan nitrogen homeostasis pathways are needed to explain the higher circulating values of citrulline found in older adults with PF\&S.

Asparagine, aspartic acid, and glutamic acid are among the six amino acids that are metabolized in resting muscles [69]. These amino acids provide the amino groups and the ammonia required for the synthesis of glutamine and alanine, which are released following protein meals and in the post-absorptive state [69]. The carbon skeletons of these metabolites may be used solely for de novo synthesis of TCA-cycle intermediates and glutamine [70]. The higher levels of asparagine, aspartic acid, and glutamic acid observed in persons with PF\&Ss may be suggestive of perturbations in muscle energy metabolism associated with muscle wasting. Interestingly, a pattern of metabolic changes accompany muscle remodeling after disuse, including energy substrate accumulation (e.g., asparagine) in atrophied muscles [71,72]. 
As opposed to EAAs, no significant differences were observed between groups in the serum levels of BCAAs. It should however be considered that the absorption of dietary proteins is influenced by several factors, which may impact their bioavailability and circulating concentrations. In particular, whether PF\&S is associated with changes in the expression of amino acid transporters and gastrointestinal physicochemical properties is presently unknown. Furthermore, the lower splanchnic extraction of BCAAs might offset subtle differences in their systemic concentrations between groups [73]. Notwithstanding, our finding on BCAAs is not consistent with previous investigations that reported changes in BCAA concentrations in relation to sarcopenia, low muscle mass, and functional limitation [21-23]. These discrepancies may be due to differences in operational definitions adopted and experimental designs among studies. In addition, heterogeneity in eating habits among participants of the different studies may contribute to the contrasting results.

The present study has some limitations that should be acknowledged. First, the study population was relatively small, and a great number of experimental variables were included in the analyses. However, the innovative analytical approach implemented in the study, based on PLS-DA plus DCV, is an ideal strategy to cope with this issue. The study sample was exclusively comprised of Caucasian individuals, which impedes generalizing the findings to other ethnic groups. Other factors that might affect circulating amino acid levels include lifestyle and eating habits [74,75]. For instance, regular participation in physical activity has been associated with reduced circulating levels of BCAAs as well as alanine and proline across a wide age spectrum [76]. Furthermore, exercise training has shown to increase the plasma levels of glycine and citrulline in overweight adults [77]. Although only people not engaged in regular exercise were enrolled in the present study, the amount of physical activity of participants was not quantified. Hence, the possible influence of physical activity on amino acid profiles in the context of PF\&S could not be established. The same applies to the possible influence of different nutritional patterns and amino acid intakes. However, as recently highlighted, differences in circulating amino acids are less marked than those between amino acid intakes [74]. The cross-sectional design of the study does not allow inference to be drawn on the time course of changes of the variables considered and on cause-effect relationships. Finally, although a fairly large number of amino acids and derivatives was assayed, it cannot be excluded that more powerful biomarkers of PF\&S might be obtained through the analysis of a larger range of biomediators.

\section{Conclusions}

In the present study, a PLS-DA-based approach allowed distinct patterns of circulating amino acids and derivatives to be identified in older persons with and without PF\&S. The pathways unveiled by this initial investigation may be used to generate new mechanistic hypotheses on the pathophysiology of PF\&S. Furthermore, the longitudinal implementation of the proposed analytical strategy could facilitate the tracking of PF\&S condition over time and the monitoring of response to treatments. This may represent a first relevant step towards the integration of specific biochemical measurements into the assessment of PF\&S, both in clinical and research settings.

Supplementary Materials: The following are available online at http:/ / www.mdpi.com/2072-6643/10/11/1691/ s1, Partial Least Squares-Discriminant Analysis; Table S1: Eligibility criteria in BIOSPHERE; Table S2: Distribution of co-morbid conditions and prevalence of use of individual drug classes in BIOSPHERE participants with and without physical frailty \& sarcopenia (PF\&S); Table S3: Serum concentrations of non-discriminant analytes in BIOSPHERE participants with and without of physical frailty \& sarcopenia (PF\&S).

Author Contributions: Conceptualization, A.P. (Anna Picca), E.M., and R.C.; methodology, A.P. (Aniello Primiano), J.G., and S.P.; software, A.B. and F.M.; validation, A.P. (Anna Picca), F.L., E.M., H.J.C.-J., and R.C.; formal analysis, A.B. and F.M.; investigation, A.P. (Anna Picca), E.M., and R.C.; resources, A.U., F.M., and R.B.; data curation, A.P. (Anna Picca), M.B., and R.C.; writing-original draft preparation, E.M. and R.C.; writing-review and editing, A.P. (Anna Picca), F.M., H.J.C.-J., J.G., and M.B.; visualization: A.P. (Aniello Primiano), J.G., and S.P.; supervision, A.U., F.L., and R.B.; funding acquisition, R.B. 
Funding: This research was funded by a grant from Fondazione Roma (NCDs Call for Proposals 2013). The work was also partly supported by a grant from the Innovative Medicines Initiative-Joint Undertaking (IMI-JU 115621), the nonprofit research foundation "Centro Studi Achille e Linda Lorenzon", and by intramural research grants from the Università Cattolica del Sacro Cuore (D3.2 2013 and D3.2 2015).

Acknowledgments: The authors thank Vincenzo Brandi, Marianna Broccatelli, Carilia Celesti, Emanuela D'Angelo, Mariaelena D'Elia, Anna Maria Martone, Giulia Savera, and Elisabetta Serafini for their invaluable help with participant screening and assessment. The authors also thank Luca Mariotti for administrative management of the BIOSPHERE project.

Conflicts of Interest: E.M., F.L., R.B. and R.C. are partners of the SPRINTT consortium, which is partly funded by the European Federation of Pharmaceutical Industries and Associations (EFPIA). The other authors declare no conflict of interest.

\section{References}

1. Dodds, R.M.; Roberts, H.C.; Cooper, C.; Sayer, A.A. The epidemiology of sarcopenia. J. Clin. Densitom. 2015, 18, 461-466. [CrossRef] [PubMed]

2. Cao, L.; Morley, J.E. Sarcopenia is recognized as an independent condition by an International Classification of Disease, Tenth Revision, Clinical Modification (ICD-10-CM) code. J. Am. Med. Dir. Assoc. 2016, 17, 675-677. [CrossRef] [PubMed]

3. Rosenberg, I.H. Sarcopenia: Origins and clinical relevance. Clin. Geriatr. Med. 2011, 27, 337-339. [CrossRef] [PubMed]

4. Landi, F.; Calvani, R.; Cesari, M.; Tosato, M.; Martone, A.M.; Ortolani, E.; Savera, G.; Salini, S.; Sisto, A.; Picca, A.; et al. Sarcopenia: An overview on current definitions, diagnosis and treatment. Curr. Protein Pept. Sci. 2018, 19, 633-638. [CrossRef] [PubMed]

5. Hirani, V.; Blyth, F.; Naganathan, V.; Le Couteur, D.G.; Seibel, M.J.; Waite, L.M.; Handelsman, D.J.; Cumming, R.G. Sarcopenia is associated with incident disability, institutionalization, and mortality in community-dwelling older men: The Concord Health and Ageing in Men project. J. Am. Med. Dir. Assoc. 2015, 16, 607-613. [CrossRef] [PubMed]

6. Marzetti, E.; Calvani, R.; Tosato, M.; Cesari, M.; Di Bari, M.; Cherubini, A.; Collamati, A.; D'Angelo, E.; Pahor, M.; Bernabei, R.; et al. Sarcopenia: An overview. Aging Clin. Exp. Res. 2017, 29, 11-17. [CrossRef] [PubMed]

7. Cesari, M.; Calvani, R.; Marzetti, E. Frailty in older persons. Clin. Geriatr. Med. 2017, 33, 293-303. [CrossRef] [PubMed]

8. Landi, F.; Calvani, R.; Cesari, M.; Tosato, M.; Martone, A.M.; Bernabei, R.; Onder, G.; Marzetti, E. Sarcopenia as the biological substrate of physical frailty. Clin. Geriatr. Med. 2015, 31, 367-374. [CrossRef] [PubMed]

9. Cesari, M.; Landi, F.; Vellas, B.; Bernabei, R.; Marzetti, E. Sarcopenia and physical frailty: Two sides of the same coin. Front. Aging Neurosci. 2014, 6, 192. [CrossRef] [PubMed]

10. Cesari, M.; Landi, F.; Calvani, R.; Cherubini, A.; Di Bari, M.; Kortebein, P.; Del Signore, S.; Le Lain, R.; Vellas, B.; Pahor, M.; et al. Rationale for a preliminary operational definition of physical frailty and sarcopenia in the SPRINTT trial. Aging Clin. Exp. Res. 2017, 29, 81-88. [CrossRef] [PubMed]

11. Marzetti, E.; Calvani, R.; Landi, F.; Hoogendijk, E.O.; Fougère, B.; Vellas, B.; Pahor, M.; Bernabei, R.; Cesari, M.; SPRINTT Consortium. Innovative Medicines Initiative: The SPRINTT project. J. Frailty Aging 2015, 4, 207-208. [CrossRef] [PubMed]

12. Cesari, M.; Marzetti, E.; Calvani, R.; Vellas, B.; Bernabei, R.; Bordes, P.; Roubenoff, R.; Landi, F.; Cherubini, A.; SPRINTT Consortium. The need of operational paradigms for frailty in older persons: The SPRINTT project. Aging Clin. Exp. Res. 2017, 29, 3-10. [CrossRef] [PubMed]

13. Calvani, R.; Marini, F.; Cesari, M.; Tosato, M.; Anker, S.D.; von Haehling, S.; Miller, R.R.; Bernabei, R.; Landi, F.; Marzetti, E.; et al. Biomarkers for physical frailty and sarcopenia: State of the science and future developments. J. Cachexia Sarcopenia Muscle 2015, 6, 278-286. [CrossRef] [PubMed]

14. Calvani, R.; Marini, F.; Cesari, M.; Tosato, M.; Picca, A.; Anker, S.D.; von Haehling, S.; Miller, R.R.; Bernabei, R.; Landi, F.; et al. Biomarkers for physical frailty and sarcopenia. Aging Clin. Exp. Res. 2017, 29, $29-34$. [CrossRef] [PubMed] 
15. Brook, M.S.; Wilkinson, D.J.; Phillips, B.E.; Perez-Schindler, J.; Philp, A.; Smith, K.; Atherton, P.J. Skeletal muscle homeostasis and plasticity in youth and ageing: Impact of nutrition and exercise. Acta Physiol. (Oxf.) 2016, 216, 15-41. [CrossRef] [PubMed]

16. Zhenyukh, O.; Civantos, E.; Ruiz-Ortega, M.; Sánchez, M.S.; Vázquez, C.; Peiró, C.; Egido, J.; Mas, S. High concentration of branched-chain amino acids promotes oxidative stress, inflammation and migration of human peripheral blood mononuclear cells via mTORC1 activation. Free Radic. Biol. Med. 2017, 104, 165-177. [CrossRef] [PubMed]

17. Yoon, M.-S. The Emerging Role of Branched-Chain Amino Acids in Insulin Resistance and Metabolism. Nutrients 2016, 8, 405. [CrossRef] [PubMed]

18. Landi, F.; Calvani, R.; Tosato, M.; Martone, A.M.; Ortolani, E.; Savera, G.; D'Angelo, E.; Sisto, A.; Marzetti, E. Protein intake and muscle health in old age: From biological plausibility to clinical evidence. Nutrients 2016, 8, 295. [CrossRef] [PubMed]

19. Pasini, E.; Corsetti, G.; Aquilani, R.; Romano, C.; Picca, A.; Calvani, R.; Dioguardi, F.S. Protein-amino acid metabolism disarrangements: The hidden enemy of chronic age-related conditions. Nutrients 2018, 10, 391. [CrossRef] [PubMed]

20. He, Q.; Yin, Y.; Zhao, F.; Kong, X.; Wu, G.; Ren, P. Metabonomics and its role in amino acid nutrition research. Front. Biosci. (Landmark Ed.) 2011, 16, 2451-2460. [CrossRef] [PubMed]

21. Lustgarten, M.S.; Price, L.L.; Chale, A.; Phillips, E.M.; Fielding, R.A. Branched chain amino acids are associated with muscle mass in functionally limited older adults. J. Gerontol. A Biol. Sci. Med. Sci. 2014, 69, 717-724. [CrossRef] [PubMed]

22. Moaddel, R.; Fabbri, E.; Khadeer, M.A.; Carlson, O.D.; Gonzalez-Freire, M.; Zhang, P.; Semba, R.D.; Ferrucci, L. Plasma biomarkers of poor muscle quality in older men and women from the Baltimore Longitudinal Study of Aging. J. Gerontol. A Biol. Sci. Med. Sci. 2016, 71, 1266-1272. [CrossRef] [PubMed]

23. Ottestad, I.; Ulven, S.M.; Øyri, L.K.L.; Sandvei, K.S.; Gjevestad, G.O.; Bye, A.; Sheikh, N.A.; Biong, A.S.; Andersen, L.F.; Holven, K.B. Reduced plasma concentration of branched-chain amino acids in sarcopenic older subjects: A cross-sectional study. Br. J. Nutr. 2018, 120, 445-453. [CrossRef] [PubMed]

24. Toyoshima, K.; Nakamura, M.; Adachi, Y.; Imaizumi, A.; Hakamada, T.; Abe, Y.; Kaneko, E.; Takahashi, S.; Shimokado, K. Increased plasma proline concentrations are associated with sarcopenia in the elderly. PLoS ONE 2017, 12, e0185206. [CrossRef] [PubMed]

25. Adachi, Y.; Ono, N.; Imaizumi, A.; Muramatsu, T.; Andou, T.; Shimodaira, Y.; Nagao, K.; Kageyama, Y.; Mori, M.; Noguchi, Y.; et al. Plasma amino acid profile in severely frail elderly patients in Japan. Int. J. Gerontol. 2018. [CrossRef]

26. Calvani, R.; Picca, A.; Marini, F.; Biancolillo, A.; Cesari, M.; Pesce, V.; Lezza, A.M.S.; Bossola, M.; Leeuwenburgh, C.; Bernabei, R.; et al. The "BIOmarkers associated with Sarcopenia and PHysical frailty in EldeRly pErsons" (BIOSPHERE) study: Rationale, design and methods. Eur. J. Intern. Med. 2018, 56, 19-25. [CrossRef] [PubMed]

27. Guralnik, J.M.; Simonsick, E.M.; Ferrucci, L.; Glynn, R.J.; Berkman, L.F.; Blazer, D.G.; Scherr, P.A.; Wallace, R.B. A short physical performance battery assessing lower extremity function: Association with self-reported disability and prediction of mortality and nursing home admission. J. Gerontol. 1994, 49, M85-M94. [CrossRef] [PubMed]

28. Studenski, S.A.; Peters, K.W.; Alley, D.E.; Cawthon, P.M.; McLean, R.R.; Harris, T.B.; Ferrucci, L.; Guralnik, J.M.; Fragala, M.S.; Kenny, A.M.; et al. The FNIH sarcopenia project: Rationale, study description, conference recommendations, and final estimates. J. Gerontol. A Biol. Sci. Med. Sci. 2014, 69, 547-558. [CrossRef] [PubMed]

29. Newman, A.B.; Simonsick, E.M.; Naydeck, B.L.; Boudreau, R.M.; Kritchevsky, S.B.; Nevitt, M.C.; Pahor, M.; Satterfield, S.; Brach, J.S.; Studenski, S.A.; et al. Association of long-distance corridor walk performance with mortality, cardiovascular disease, mobility limitation, and disability. JAMA 2006, 295, 2018-2026. [CrossRef] [PubMed]

30. Barker, M.; Rayens, W. Partial least squares for discrimination. J. Chemom. 2003, 17, 166-173. [CrossRef]

31. Wold, S.; Martens, H.; Wold, H. The multivariate calibration problem in chemistry solved by the PLS method. In Matrix Pencils; Lecture Notes in Mathematics; Springer: Berlin, Germany, 1983; Volume 973, pp. $286-293$. ISBN 978-3-540-11983-8. 
32. Westerhuis, J.A.; Hoefsloot, H.C.J.; Smit, S.; Vis, D.J.; Smilde, A.K.; van Velzen, E.J.J.; van Duijnhoven, J.P.M.; van Dorsten, F.A. Assessment of PLSDA cross validation. Metabolomics 2008, 4, 81-89. [CrossRef]

33. Szymańska, E.; Saccenti, E.; Smilde, A.K.; Westerhuis, J.A. Double-check: Validation of diagnostic statistics for PLS-DA models in metabolomics studies. Metabolomics 2012, 8, 3-16. [CrossRef] [PubMed]

34. Smit, S.; van Breemen, M.J.; Hoefsloot, H.C.J.; Smilde, A.K.; Aerts, J.M.F.G.; de Koster, C.G. Assessing the statistical validity of proteomics based biomarkers. Anal. Chim. Acta 2007, 592, 210-217. [CrossRef] [PubMed]

35. Wu, G. Amino acids: Metabolism, functions, and nutrition. Amino Acids 2009, 37, 1-17. [CrossRef] [PubMed]

36. Kouchiwa, T.; Wada, K.; Uchiyama, M.; Kasezawa, N.; Niisato, M.; Murakami, H.; Fukuyama, K.; Yokogoshi, H. Age-related changes in serum amino acids concentrations in healthy individuals. Clin. Chem. Lab. Med. 2012, 50, 861-870. [CrossRef] [PubMed]

37. Cruz-Jentoft, A.J.; Kiesswetter, E.; Drey, M.; Sieber, C.C. Nutrition, frailty, and sarcopenia. Aging Clin. Exp. Res. 2017, 29, 43-48. [CrossRef] [PubMed]

38. Calvani, R.; Miccheli, A.; Landi, F.; Bossola, M.; Cesari, M.; Leeuwenburgh, C.; Sieber, C.C.; Bernabei, R.; Marzetti, E. Current nutritional recommendations and novel dietary strategies to manage sarcopenia. J. Frailty Aging 2013, 2, 38-53. [CrossRef] [PubMed]

39. Matsuo, Y.; Greenberg, D.M. Metabolic formation of homoserine and alpha-aminobutyric acid from methionine. J. Biol. Chem. 1955, 215, 547-554. [PubMed]

40. Haschke-Becher, E.; Kainz, A.; Bachmann, C. Reference values of amino acids and of common clinical chemistry in plasma of healthy infants aged 1 and 4 months. J. Inherit. Metab. Dis. 2016, 39, 25-37. [CrossRef] [PubMed]

41. Kar, S.K.; Jansman, A.J.M.; Schokker, D.; Kruijt, L.; Harms, A.C.; Wells, J.M.; Smits, M.A. Amine metabolism is influenced by dietary protein source. Front. Nutr. 2017, 4, 41. [CrossRef] [PubMed]

42. Ducker, G.S.; Rabinowitz, J.D. One-carbon metabolism in health and disease. Cell Metab. 2017, $25,27-42$. [CrossRef] [PubMed]

43. Suh, E.; Choi, S.-W.; Friso, S. One-carbon metabolism: An unsung hero for healthy aging. Mol. Basis Nutr. Aging 2016, 513-522. [CrossRef]

44. Mudd, S.H.; Ebert, M.H.; Scriver, C.R. Labile methyl group balances in the human: The role of sarcosine. Metabolism 1980, 29, 707-720. [CrossRef]

45. Allen, R.H.; Stabler, S.P.; Lindenbaum, J. Serum betaine, $N, N$-dimethylglycine and $N$-methylglycine levels in patients with cobalamin and folate deficiency and related inborn errors of metabolism. Metabolism 1993, 42, 1448-1460. [CrossRef]

46. Walters, R.O.; Fontana, L.; Kurland, I.; Diaz, A.; Arias-Perez, E.; Cuervo, A.; Promislow, D.; Huffman, D. Sarcosine is uniquely modulated by aging and dietary restriction in rodents and humans. Innov. Aging 2017, 1, 1208-1209. [CrossRef]

47. Marzetti, E.; Calvani, R.; Lorenzi, M.; Tanganelli, F.; Picca, A.; Bossola, M.; Menghi, A.; Bernabei, R.; Landi, F. Association between myocyte quality control signaling and sarcopenia in old hip-fractured patients: Results from the Sarcopenia in HIp FracTure (SHIFT) exploratory study. Exp. Gerontol. 2016, 80, 1-5. [CrossRef] [PubMed]

48. Calvani, R.; Joseph, A.-M.; Adhihetty, P.J.; Miccheli, A.; Bossola, M.; Leeuwenburgh, C.; Bernabei, R.; Marzetti, E. Mitochondrial pathways in sarcopenia of aging and disuse muscle atrophy. Biol. Chem. 2013, 394, 393-414. [CrossRef] [PubMed]

49. Picca, A.; Calvani, R.; Lorenzi, M.; Menghi, A.; Galli, M.; Vitiello, R.; Randisi, F.; Bernabei, R.; Landi, F.; Marzetti, E. Mitochondrial dynamics signaling is shifted toward fusion in muscles of very old hip-fractured patients: Results from the Sarcopenia in HIp FracTure (SHIFT) exploratory study. Exp. Gerontol. 2017, 96, 63-67. [CrossRef] [PubMed]

50. Picca, A.; Calvani, R.; Bossola, M.; Allocca, E.; Menghi, A.; Pesce, V.; Lezza, A.M.S.; Bernabei, R.; Landi, F.; Marzetti, E. Update on mitochondria and muscle aging: All wrong roads lead to sarcopenia. Biol. Chem. 2018, 399, 421-436. [CrossRef] [PubMed]

51. Vance, J.E. Phosphatidylserine and phosphatidylethanolamine in mammalian cells: Two metabolically related aminophospholipids. J. Lipid Res. 2008, 49, 1377-1387. [CrossRef] [PubMed] 
52. Rockenfeller, P.; Koska, M.; Pietrocola, F.; Minois, N.; Knittelfelder, O.; Sica, V.; Franz, J.; Carmona-Gutierrez, D.; Kroemer, G.; Madeo, F. Phosphatidylethanolamine positively regulates autophagy and longevity. Cell Death Differ. 2015, 22, 499-508. [CrossRef] [PubMed]

53. van der Veen, J.N.; Kennelly, J.P.; Wan, S.; Vance, J.E.; Vance, D.E.; Jacobs, R.L. The critical role of phosphatidylcholine and phosphatidylethanolamine metabolism in health and disease. Biochim. Biophys. Acta 2017, 1859, 1558-1572. [CrossRef] [PubMed]

54. Selathurai, A.; Kowalski, G.M.; Burch, M.L.; Sepulveda, P.; Risis, S.; Lee-Young, R.S.; Lamon, S.; Meikle, P.J.; Genders, A.J.; McGee, S.L.; et al. The CDP-ethanolamine pathway regulates skeletal muscle diacylglycerol content and mitochondrial biogenesis without altering insulin sensitivity. Cell Metab. 2015, 21, 718-730. [CrossRef] [PubMed]

55. Huxtable, R.J. Physiological actions of taurine. Physiol. Rev. 1992, 72, 101-163. [CrossRef] [PubMed]

56. Conte Camerino, D.; Tricarico, D.; Pierno, S.; Desaphy, J.-F.; Liantonio, A.; Pusch, M.; Burdi, R.; Camerino, C.; Fraysse, B.; De Luca, A. Taurine and skeletal muscle disorders. Neurochem. Res. 2004, 29, 135-142. [CrossRef] [PubMed]

57. De Luca, A.; Pierno, S.; Camerino, D.C. Taurine: The appeal of a safe amino acid for skeletal muscle disorders. J. Transl. Med. 2015, 13, 243. [CrossRef] [PubMed]

58. Lambert, I.H.; Kristensen, D.M.; Holm, J.B.; Mortensen, O.H. Physiological role of taurine-from organism to organelle. Acta Physiol. (Oxf.) 2015, 213, 191-212. [CrossRef] [PubMed]

59. Ito, T.; Yoshikawa, N.; Inui, T.; Miyazaki, N.; Schaffer, S.W.; Azuma, J. Tissue depletion of taurine accelerates skeletal muscle senescence and leads to early death in mice. PLoS ONE 2014, 9, e107409. [CrossRef] [PubMed]

60. Scicchitano, B.M.; Sica, G. The beneficial effects of taurine to counteract sarcopenia. Curr. Protein Pept. Sci. 2018, 19, 673-680. [CrossRef] [PubMed]

61. Tomás-Loba, A.; Bernardes de Jesus, B.; Mato, J.M.; Blasco, M.A. A metabolic signature predicts biological age in mice. Aging Cell 2013, 12, 93-101. [CrossRef] [PubMed]

62. Breuillard, C.; Cynober, L.; Moinard, C. Citrulline and nitrogen homeostasis: An overview. Amino Acids 2015, 47, 685-691. [CrossRef] [PubMed]

63. Papadia, C.; Osowska, S.; Cynober, L.; Forbes, A. Citrulline in health and disease. Review on human studies. Clin. Nutr. 2017. [CrossRef] [PubMed]

64. Le Plénier, S.; Walrand, S.; Noirt, R.; Cynober, L.; Moinard, C. Effects of leucine and citrulline versus non-essential amino acids on muscle protein synthesis in fasted rat: A common activation pathway? Amino Acids 2012, 43, 1171-1178. [CrossRef] [PubMed]

65. Martone, A.M.; Lattanzio, F.; Abbatecola, A.M.; Carpia, D.L.; Tosato, M.; Marzetti, E.; Calvani, R.; Onder, G.; Landi, F. Treating sarcopenia in older and oldest old. Curr. Pharm. Des. 2015, 21, 1715-1722. [CrossRef] [PubMed]

66. Sarwar, G.; Botting, H.G.; Collins, M. A comparison of fasting serum amino acid profiles of young and elderly subjects. J. Am. Coll. Nutr. 1991, 10, 668-674. [CrossRef] [PubMed]

67. Pitkänen, H.T.; Oja, S.S.; Kemppainen, K.; Seppä, J.M.; Mero, A.A. Serum amino acid concentrations in aging men and women. Amino Acids 2003, 24, 413-421. [CrossRef] [PubMed]

68. Chaleckis, R.; Murakami, I.; Takada, J.; Kondoh, H.; Yanagida, M. Individual variability in human blood metabolites identifies age-related differences. Proc. Natl. Acad. Sci. USA 2016, 113, 4252-4259. [CrossRef] [PubMed]

69. Wagenmakers, A.J. Muscle amino acid metabolism at rest and during exercise: Role in human physiology and metabolism. Exerc. Sport Sci. Rev. 1998, 26, 287-314. [CrossRef] [PubMed]

70. Wagenmakers, A.J. Protein and amino acid metabolism in human muscle. Adv. Exp. Med. Biol. 1998, 441, 307-319. [CrossRef] [PubMed]

71. Stein, T.P.; Wade, C.E. Metabolic consequences of muscle disuse atrophy. J. Nutr. 2005, 135, 1824S-1828S. [CrossRef] [PubMed]

72. Ilaiwy, A.; Quintana, M.T.; Bain, J.R.; Muehlbauer, M.J.; Brown, D.I.; Stansfield, W.E.; Willis, M.S. Cessation of biomechanical stretch model of $\mathrm{C} 2 \mathrm{C} 12$ cells models myocyte atrophy and anaplerotic changes in metabolism using non-targeted metabolomics analysis. Int. J. Biochem. Cell Biol. 2016, 79, 80-92. [CrossRef] [PubMed]

73. Soultoukis, G.A.; Partridge, L. Dietary protein, metabolism, and aging. Annu. Rev. Biochem. 2016, 85, 5-34. [CrossRef] [PubMed] 
74. Schmidt, J.A.; Rinaldi, S.; Scalbert, A.; Ferrari, P.; Achaintre, D.; Gunter, M.J.; Appleby, P.N.; Key, T.J.; Travis, R.C. Plasma concentrations and intakes of amino acids in male meat-eaters, fish-eaters, vegetarians and vegans: A cross-sectional analysis in the EPIC-Oxford cohort. Eur. J. Clin. Nutr. 2016, 70, 306-312. [CrossRef] [PubMed]

75. Fukai, K.; Harada, S.; Iida, M.; Kurihara, A.; Takeuchi, A.; Kuwabara, K.; Sugiyama, D.; Okamura, T.; Akiyama, M.; Nishiwaki, Y.; et al. Metabolic profiling of total physical activity and sedentary behavior in community-dwelling men. PLoS ONE 2016, 11, e0164877. [CrossRef] [PubMed]

76. Kujala, U.M.; Mäkinen, V.-P.; Heinonen, I.; Soininen, P.; Kangas, A.J.; Leskinen, T.H.; Rahkila, P.; Würtz, P.; Kovanen, V.; Cheng, S.; et al. Long-term leisure-time physical activity and serum metabolome. Circulation 2013, 127, 340-348. [CrossRef] [PubMed]

77. Glynn, E.L.; Piner, L.W.; Huffman, K.M.; Slentz, C.A.; Elliot-Penry, L.; AbouAssi, H.; White, P.J.; Bain, J.R.; Muehlbauer, M.J.; Ilkayeva, O.R.; et al. Impact of combined resistance and aerobic exercise training on branched-chain amino acid turnover, glycine metabolism and insulin sensitivity in overweight humans. Diabetologia 2015, 58, 2324-2335. [CrossRef] [PubMed]

(C) 2018 by the authors. Licensee MDPI, Basel, Switzerland. This article is an open access article distributed under the terms and conditions of the Creative Commons Attribution (CC BY) license (http://creativecommons.org/licenses/by/4.0/). 